\title{
Mujeres en altos cargos en minería en Chile. Agencia y tensiones de género
}

\section{Women in senior positions in mining in Chile. Agency and gender tensions}

\author{
Pamela Caro (i) https://orcid.org/0000-0001-8177-9295 \\ Universidad Santo Tomás, Chile, pamelacaro1@santotomas.cl
}

Helena Román (i) https://orcid.org/0000-0002-3459-0411

UniversidadSanto Tomás, Chile,hroman@santotomas.cl

Lorena Armijo $\mathbb{D}^{\mathbb{D}}$ https://orcid.org/0000-0003-4980-9524

Universidad Santo Tomás, Chile, lorenaarmijoga@santotomas.cl

\begin{abstract}
The article aims to understand the relation between gender tensions due to the countercultural situation of women in senior positions in Chilean mining industry, and the strategies and capacities to face them, seen as agency. By using a qualitative methodology, we analyze 13 life stories and conclude that, even in contexts of gender inequality, upward social mobility trajectories question the traditional order. The main tensions are related to family roles, power and recognition, cultural representations of the environment and redefinition of identities. The pre-eminently individual strategies refer to more egalitarian conjugal arrangements; continuity in education; identity based on action, success and achievements; and, a combination of strong character and self-confidence together with loving care, rejecting masculinized leaderships.
\end{abstract}

Key words: women in senior roles, mining, gender tensions, trajectories, capacity for agency.

Resumen: El artículo busca comprender la relación entre las tensiones de género por la posición contracultural de mujeres en cargos de

*Autor para

correspondencia:

Pamela Caro

Recepción: 12/05/2020

Aprobación: 05/12/2020

Publicación: 25/02/21 jefatura en minería en Chile y las estrategias y capacidades para enfrentarlas, entendidas como agencia. $\mathrm{Al}$ analizar cualitativamente 13 relatos de vida, concluimos que aun en contextos de desigualdad de género, trayectorias ascendentes, interrogan al orden tradicional. Las principales tensiones se presentan en los roles familiares, poder y reconocimiento, representaciones culturales del medio y redefinición de identidades. Las estrategias, preeminentemente individuales, refieren a: arreglos conyugales más igualitarios; continuidad de estudios; identidad basada en la acción, éxito y logros; conjugar fortaleza de carácter y seguridad en sí mismas con trato amoroso, rechazando la masculinización de los liderazgos.

Palabras clave: mujeres en altos cargos, minería, tensiones de género, trayectorias, capacidad de agencia. 


\section{Introducción $^{1}$}

La minería en Chile es una industria que históricamente excluyó a las mujeres o las relegó a funciones feminizadas -administrativas, alimentación o aseo- (Angelcos, 2015). Recién en la última década comienza a incrementarse con lentitud su participación en labores propiamente mineras, favorecida, al igual que en otros países, por la necesidad de ampliar dotaciones (Soraire et al., 2013). En la actualidad, los datos muestran que las mujeres representan $8,9 \%$ de las dotaciones ${ }^{2}$ (3,3\% en 2003), siendo el promedio en cargos de directoras/gerentas 11,9\%, subgerentas/superintendentas 11,1\% y jefaturas 8,4\% (CCM, 2019). En el país se ha problematizado empíricamente en torno a las barreras de género de la industria, estructura y cultura organizacional, así como en la debilidad de medidas de conciliación de familia y trabajo (Salinas et al., 2012; Leiva y Comelin, 2015). Se concluye que la cultura minera ha generado una jerarquía dominada por hombres, quienes garantizan sus privilegios mediante la diferenciación funcional del trabajo por sexo (Tallichet, 1995, en Salinas et al., 2012: 141), la cual seguiría acompañada del refuerzo simbólico a la exclusión femenina por medio de mitos y supersticiones (Campos et al., 2016). Salvo un estudio sobre liderazgo masculino en entornos mineros (Salinas y Cordero, 2016), no se ha profundizado en la experiencia de mujeres insertas en posiciones de poder en faenas y operaciones, evidenciando que las tensiones de género en este nicho del mercado laboral se hacen presentes incluso desde la investigación académica, que relega el estudio de las mujeres en posiciones diferentes a las que históricamente han ocupado.

Este artículo sigue la línea de investigación sobre género, trabajo y minería, como sector productivo masculinizado. Se inscribe en una investigación más amplia, cuyo objetivo es conocer las implicancias subjetivas del ingreso de mujeres en posiciones de poder de la cadena de valor principal (extracción, procesamiento y mantenimiento). Con el uso del concepto de agencia (Sen, 1999, 2009) como lente de aproximación a trayectorias laborales, y la categoría de género (Scott, 2008) para comprender el entramado

1 E 1 artículo presenta resultados del Proyecto ANID/FONDECYT/REGULAR/ $\mathrm{N}^{\circ} 1180590$, por lo que agradecemos a ANID/FONDECYT (Agencia Nacional de Investigación y Desarrollo/Fondo Nacional de Ciencia y Tecnología).

2 Labaja participación de las mujeres en la minería también ocurre en otros países productores como Australia o Canadá, con tasas de participación de $17 \%$ y $18 \%$, respectivamente; pero se debe considerar que en dichos países la PEA global femenina es de $72 \%$ y $75 \%$, respectivamente; más alta de la registrada en Chile, que es sólo de 48\% (CCM, 2019). 
de las relaciones sociales de poder entre hombres y mujeres -mediante un abordaje cualitativo-, exploramos experiencias que evidenciarían cuestionamientos al orden de género tradicional y fisuras al "techo de cristal" (Burin, 1990). ${ }^{3}$ En la investigación privilegiamos la observación de lo que se moviliza desde una posición laboral transgresora, y no sólo desde la perspectiva de la subordinación que permitirá probar "lo que ya sabemos" (Tarrés, 2001b).

Las principales preguntas de investigación que articularon el artículo son: ¿cómo las mujeres en puestos no tradicionales a su sexo, de jefatura, gerencia y dirección, en una industria masculinizada como la minería chilena, desarrollaron trayectorias laborales, afrontando las tensiones de género surgidas en contextos hostiles a la igualdad de género? y ¿qué estrategias, como capacidades de agencia y recursos, han sido y son desplegadas en el ejercicio de sus cargos, posibles de apreciar a través de sus trayectorias personales y ocupacionales? De manera complementaria, nos preguntamos: ¿qué papel juegan las instituciones (empresas e instancias gubernamentales), como parte del espacio mesosocial, en la modificación/perpetuación de hostilidad de género, alentando o limitando las posibilidades de agencia en sus trayectorias microsociales? Responderemos a esta interrogante entretejiendo el análisis.

La principal hipótesis de investigación que sustentamos es que trayectorias laborales femeninas contrarias a los mandatos normativos, aun en contextos de desigualdad y hostilidad hacia las mujeres, interrogan el orden tradicional de género. La hipótesis específica del artículo es que trayectorias educativas en carreras no tradicionales -sorteando discriminaciones de género tempranamente-, con proyecciones de continuidad de estudios, modelos marentales emancipatorios, arreglos familiares más igualitarios en el presente y liderazgos colaborativos, son factores que inciden positivamente en mujeres que han logrado posicionarse en puestos de liderazgo en minería, iniciando transformaciones en las estructuras tradicionales de poder. En 3 La metáfora "techo de cristal" (Burin, 1990) ha sido útil para expresar el límite invisible, a veces "secreto", en las carreras laborales femeninas ascendentes, difícil de traspasar, y que obstaculiza desarrollos profesionales. Se construye no a partir de leyes, dispositivos sociales o códigos explícitos que impongan limitaciones, sino que sus cimientos son sutiles y están anclados a la naturalización, que los hacen difíciles de detectar. Los conforman aspectos culturales y estructurales. El concepto ha sido descrito por la sociología y economía anglosajona, que en los años ochenta buscó explicar la subrepresentación de las mujeres en puestos altos (Holloway, 1993; Davidson y Cooper, 1992; Morrison, 1992, citadas en Burin, 2004). En su dimensión objetiva es una realidad discriminatoria, y en su dimensión subjetiva impone detención y retroceso en proyectos laborales (Burin, 2004). 
dicho proceso desarrollan recursos personales y estrategias relacionales que las han llevado a soslayar discriminaciones y barreras, impulsando -en tanto sujetos sociales agenciados- no sólo transformaciones micro, sino también macrosociales (Elias, 1990).

Mediante el análisis de 13 relatos de vida sobre trayectorias personales y laborales, ${ }^{4}$ comprendidas como experiencias intersubjetivas de mujeres que ejercen cargos altos en mineras del norte y centro de Chile, estudiamos un proceso en el cual, contradiciendo identidades estereotipadas, tomaron decisiones, desarrollaron trayectorias -educacionales, formativas y laborales- individualizadas, en tanto constituyeron elecciones autónomas y se incorporaron a una industria masculinizada, ocupando cargos pioneros a su sexo.

\section{Antecedentes}

\section{Las mujeres en la industria minera chilena}

Recién en 1996 se derogó la Ley 18.620, que prohibía el trabajo minero subterráneo de las mujeres, desafiando creencias en torno a que su presencia podría traer mala suerte a la actividad o "hacer enojar a la mina". Investigaciones previas evidencian barreras de género que atañen a la cultura organizacional, pues durante décadas existió una jerarquía interna organizada en torno a códigos masculinos (Salinas y Romani, 2016; Salinas y Cordero, 2016), que se tradujeron en un contexto hostil, prácticas y discursos de discriminación (Jiménez et al., 2014; Angelcos e Ísola, 2017), y en su versión más extrema en experiencias de acoso sexual y laboral (Caro et al., 2019).

Las mujeres se enfrentarían a definiciones de género construidas de acuerdo con una determinada distribución de roles y expectativas que establecería quién es quién y cómo debe actuar (Angelcos, 2015). El ambiente en faenas es extremadamente duro. Los riesgos para la salud y las condiciones ambientales extremas indujeron a pensar a la industria con constricciones legítimas que la concibieran como un trabajo "para hombres" (Ayala, 2012).

4 Para la investigación más amplia, se entrevistó a un total de 35 mujeres (13 ejecutivas/ directivas, 11 operarias pioneras y 11 dirigentas sindicales).

5 La presencia femenina podría esconder la veta, o hacer que se ponga celosa y se produzcan accidentes. Este fenómeno no es exclusivamente chileno, pues en Bolivia existe como variante del mito la idea de que la mina o "el tío" se puede excitar con la entrada de mujeres, al punto de generar algún desastre (Ruiz, 2003). 
Al polvo, altura geográfica, paisaje agreste y temperaturas extremas, se suma el aislamiento, baja conectividad y desconexión familiar. La organización del trabajo incluye movilidad geográfica, jornadas de trabajo extensas, vida en campamentos y sistemas de turnos, que dan lugar a experiencias de dislocación y desajuste de la vida cotidiana (Mayes, 2019). Esto ha vuelto más compleja la inserción de mujeres, dado que cargan con el peso histórico del trabajo reproductivo; además, las menores posibilidades de conciliación entre trabajo y maternidad desmotivan la participación y exacerba las tensiones. De acuerdo con el Instituto Nacional de Estadísticas (2018), la participación de trabajadoras en minería se concentra en mujeres de entre 25 y 44 años. Tienen una edad promedio menor a los hombres y mayores niveles de educación y profesionalización (Caro et al., 2019). La sobrecalificación es percibida como una forma de injusticia (Angelcos e Ísola, 2017). Por su parte, la maternidad es vista como interferencia y tiene un peso simbólico negativo en las trayectorias laborales, principalmente asociada a la extensión de licencias médicas, por la percepción del aumento de costos laborales, aun cuando se concluye que son reducidos (Riquelme, 2011) y de cargo estatal (subsidio). Estudios internacionales han acuñado el concepto "muro de la maternidad", pues las condiciones de trabajo y estereotipos culturales generan el sesgo de que las mujeres con hijos/as pequeños no pueden ser buenas profesionales en esta industria, mostrando una abierta discriminación (Minerals Council of Australia, 2013).

Este mercado evidencia segregación vertical, pues las mujeres tienen menores posibilidades de ocupar cargos superiores, dada su menor experiencia y especialización. Para ascender en jerarquía, es necesario cumplir requisitos, en menor proporción en ellas, como años de servicios, evaluaciones de desempeño, acreditaciones teóricas y evaluaciones psicológicas (Díaz, 2014). Suelen esperar más tiempo para postular a una promoción laboral, y la brecha salarial aumenta en los cargos altos (Minerals Council of Australia, 2013). $\mathrm{Al}$ mismo tiempo existe segregación horizontal. Los hombres se concentran en cargos de operadores (34\%) y mantenedores (30\%), mientras que ellas en los cargos profesionales (25\%) y de soporte administrativo (23\%) (CCM, 2019). Angelcos (2015: 10) identificó dos elementos que constituyen ejes vertebradores de discursos de segregación de género en minería. El primero está asociado a las diferencias de fuerza física, que convierte a los hombres en "los capacitados" y elegibles, ignorando las posibilidades de las nuevas tecnologías. El segundo está asociado a la maternidad.

Frente a un contexto históricamente adverso, en la actualidad, empresas de la gran minería sostienen una declaración favorable sobre la equidad y 
han desarrollado de manera incipiente algunos programas; $80 \%$ ha señalado objetivos de retención y atracción, enmarcados en estrategias de diversidad, pero sólo 35\% cuenta con políticas explícitas para la contratación de mujeres. Las acciones incluyen talleres de sensibilización y el otorgamiento de posibilidades para que las mujeres asistan a programas de liderazgo y teletrabajo (CCM, 2019), actualmente masificado por los efectos de la pandemia covid-19. Desde el nivel gubernamental, la principal iniciativa es la promoción de una norma voluntaria: Norma Chilena 3262 (2012), de igualdad de género y conciliación, que a través de un sistema de gestión exige una serie de requisitos para alcanzar certificación y sello (Caro et al., 2019). Sin embargo, ha tenido una limitada penetración, pues hasta ahora sólo ha certificado una minera estatal (y sus 10 filiales) y otra privada. Se colige que la coyuntura política y social es más favorable que en el pasado para fomentar la asunción de mujeres en altos cargos, pero no es una relación lineal. Tampoco se ha medido cómo estas acciones inciden en la agencia individual de las mujeres.

\section{Género, orden social y tensiones en las trayectorias}

Para abordar las preguntas de investigación, utilizaremos como marco teórico la perspectiva de género, desde el feminismo crítico (Scott, 2008; Héritier, 2007; Fraser, 2011; Tarrés, 2001a, 2001b y 2013), así como la categoría de agencia desde el enfoque de las capacidades (Sen, 1985, 1999 y 2009; Nussbaum, 2014; Cejudo, 2007; Otano, 2015; Keleher, 2014), problematizando los conceptos de libertad y logro de agencia. La base teórica de la perspectiva de género nos lleva a comprender que las relaciones entre sujetos sexuados son formas primarias de vínculos significantes de poder (Scott, 2008). Desde este enfoque, roles de hombres y mujeres son interpretados como construcción social de base material e ideológica, comprensible históricamente, rompiendo explicaciones biologizantes y modelos universales. Así, los comportamientos genéricos y la división sexual del trabajo son un asunto de aprendizaje material y simbólico adquirido por la socialización (Héritier, 2007), donde se desprenden comportamientos adecuados y relaciones apropiadas (Rubin, 1975), bajo un orden tradicional, en marcos de dominación, anclado en mandatos culturales naturalizados.

Fraser (2011) sostiene que el género es una diferenciación económicopolítica, pero también de valoración cultural, mediante la cual se configuran formas de hacer, experiencias y representaciones. Desde esa perspectiva, no es difícil ver cómo el género y el orden social son parte del mismo engranaje, donde convergen prácticas que regulan el funcionamiento de los sujetos 
y, simultáneamente, los habilitan para operar en ellas (Rebolledo y Valdés, 2018).

El género, desde una epistemología feminista crítica, nos permite problematizar las trayectorias ocupacionales de las mujeres en la minería, más allá de verlas sólo como víctimas (Lahiri-Dutt, 2012). Vivimos actualmente en un mundo de cambios normativos introducidos por el proceso de emancipación de mujeres que han comenzado a moverse, aun cuando no se les facilite necesariamente el camino. Esta estrategia discursiva puede contribuir a ilustrar los movimientos irreversibles por cuya agenda cruza buena parte de las actuales tensiones civilizatorias (Valcárcel, 2009). Observar la posición de mujeres en altos cargos en minería desde el lente del cuestionamiento al orden de género tradicional, posibilitaría el desarrollo de acciones reflexivas orientadas a la transformación de condiciones y relaciones de poder (Tarrés, 2001a).

Para dar cuenta del doble proceso de apropiación del mundo social desde la experiencia de las mujeres, la propuesta de Tarrés (2007, en Olivera, 2008) consiste en descomponer analíticamente su experiencia en dos niveles: 1) el movimiento histórico-estructural, que comprende procesos macrosociales que rompen patrones de reproducción femenina, y 2) el movimiento histórico-coyuntural constituido por factores que influyen en las biografías de las mujeres para redefinir sus identidades que, aunque macro históricamente han sido subordinadas, comprende microcambios que cruzan individualidades y se reflejan en acciones, intercambios y negociaciones de significados (Olivera, 2008).

Usamos el concepto de tensiones para expresar reacomodos o cambios parciales, a partir de la participación femenina en posiciones no tradicionales, al considerar que el hecho de que las mujeres ocupen altos cargos mineros responde a una situación contracultural; por ende, no es inocua y movilizaría identidades, arreglos tradicionales y la división sexual del trabajo. En las identidades femeninas permite abordar una mirada a las experiencias desde la noción de fluido, entendida como el proceso de cambio no lineal, en el cual -de manera heterogénea- se conjuga subordinación (histórica) con experiencias de actoría contingentes que, como señala Tarrés (2001b), se construye "poco a poco" y se elaboran cotidianamente.

Al contar con pocos modelos referentes para identificarse en minería, se trataría de trayectorias autoconstruidas, elaborando identidades "desde cero", pioneras, en diálogo crítico con el repertorio de roles disponibles que circulan entre sus pares hombres (Angelcos, 2015). Estudios previos han corroborado que la participación de las mujeres en la industria ha implicado 
posibilidades de llevar adelante proyectos de vida y conquistar un espacio público, que la literatura establece como sin retorno por sus cambios emancipatorios, pudiendo encontrar en dicha experiencia reconocimiento y visibilidad (Díaz, 2014).

Algunos estudios internacionales han evidenciado modificaciones de rol e identidad femenina, interrogando el sistema de género por una nueva subjetividad femenina, destacando la capacidad de superación y tenacidad (Jenkins, 2014). Ostentar un alto cargo otorgaría estatus y reconocimiento en la escena de un conflicto público, con una voz que se considera novedosa (Soraire et al., 2013). El concepto de trayectorias se usará desde el registro y análisis de posiciones sucesivas, ya que permite recoger cambios en las historias, recorridos en procesos de ascenso, poniéndose en juego recursos sociales y reacciones agenciales como estrategias (De Certeau, 2000, citado en Madero y Mora, 2011).

\section{La categoría de agencia desde el enfoque teórico de las capacidades}

Para observar los procesos que las propias mujeres -como sujeto (Tarrés, 2001a) - protagonizan, optamos por la perspectiva de las capacidades de agencia $^{6}$ (Sen, 1999), que al abordarla en diálogo con el enfoque de la justicia (Sen, 2009) no desconoce la existencia de condiciones de desigualdad estructural de género a corregir, en cuya reproducción intervienen por acción u omisión instancias mesosociales como el Estado y las propias empresas; pero enfatiza en la capacidad activa de las mujeres, de participar en procesos, que otros han llamado de transformación "desde abajo" o desde sus protagonistas (De Sousa Santos y Rodríguez, 2007).

La definición de agencia desde el enfoque de las capacidades (Sen, 1985) describe lo que una mujer es libre de hacer, ser y lograr en la búsqueda de metas que considera importantes. Es poder llevar adelante acciones que producen cambios según objetivos valorados, representando la habilidad para actuar (Alkire, 2009, citado en Leiva, 2015), y a su vez refleja la libertad para elegir entre diferentes maneras de vivir (Cejudo, 2007). Sen (1999) propone un análisis desde dos dimensiones interrelacionadas: 1) la idea de bienestar, condición de ventaja desde la dimensión personal; y 2) agencia, en tanto búsqueda de metas valoradas personales o intención y voluntad deliberada para alcanzarlas, siendo la que permite emprender acciones que produzcan cambios según propósito y obligaciones, de acuerdo con la concepción del "bien" personal.

6 La propuesta teórica original fue formulada por Sen (1985), como una opción al enfoque tradicional de la economía del bienestar. 
La agencia personal (Nussbaum, 2014), como capacidad para actuar o hacer, problematizada desde una perspectiva relacional y atravesada por el logro y libertad, necesita respeto y protección por parte de individuos, pero también de instituciones. En este sentido, el "logro de agencia" se refiere a la realización de metas y objetivos escogidos a perseguir, y "libertad de agencia", a las posibilidades de conseguir lo que se quiere lograr, limitadas por las oportunidades sociales, económicas y políticas (Keleher, 2014) del entorno. En este análisis, principalmente el Estado, en cada sociedad, puede desempeñar un papel en la inhibición o motivación para la agencia individual. En Chile, aún es una tarea pendiente en materia de igualdad de género en altos cargos, pues los incentivos públicos no han sido efectivos para acompañar las transformaciones en los imaginarios culturales (PNUD, 2019) y conducir a que más mujeres construyan trayectorias agenciadas.

Se ejerce agencia cuando se decide por sí misma, cuando se actúa con autonomía, propósito y plena conciencia de su valor. La agencia de las mujeres se configuraría cuando las vuelve protagonistas, pero también cuando resuelve conflictos y aborda ámbitos donde experimenten discriminación de género; pues en dicho proceso se pueden convertir en iniciadoras de transformaciones sociales (Forero, 2012). El desarrollo de la libertad de agencia debiera comprenderse en medio de una trama de relaciones, con conflictos de intenciones y voluntades (Otano, 2015; Leiva, 2015). Por ende, el centro de atención no queda sólo en la capacidad de elección individual, sino también en las formas de solidaridad que faciliten la expansión de capacidades (Otano, 2015). Así aparecería agencia y tensiones de género de manera simultánea, evidenciando las posibilidades de trastocar relaciones de poder (Tarrés, 2013), el orden tradicional y constituirse en palancas de cambio (Héritier, 2007) individuales o colectivas.

\section{Metodología}

La investigación recurrió a un paradigma interpretativo, sustentado en una epistemología hermenéutica y diseño cualitativo (Ruiz, 2003), utilizando como principal técnica de recolección de información los relatos de vida, con entrevistas en profundidad en uno o dos encuentros. Esto permitió recoger experiencias e historias que representan la posibilidad de recuperar sentidos vinculados a lo vivido, y a su vez, vislumbrar significaciones y construir un saber denso (Kornblit, 2004). Las entrevistas se llevaron a cabo cara a cara, lo cual posibilitó acceder a subjetividades, profundizar en la realidad social (Vela, 2004) y comprender perspectivas expresadas con sus propias palabras. 
En el estudio participaron 13 mujeres que tuvieran como principal atributo ocupar cargos de gerentas, directoras, superintendentas o jefas de planta, de preferencia en la cadena de valor primordial (extracción, mantenimiento, procesamiento) de faenas mineras del país. Se accedió a ellas mediante la estrategia de bola de nieve (Ruiz, 2003), a partir de contactos iniciales con informantes clave de la industria generados en investigaciones previas, quienes facilitaron el acceso a dicho perfil. Las entrevistas se realizaron durante 2019. Sus perfiles profesionales son ingenieras químicas y/o metalúrgicas, industriales, en minas y comerciales -sólo una es profesional de las ciencias sociales-, que trabajan en faenas del norte y centro del país (salvo dos que ejercen en la capital).

Para el resguardo de la investigación se solicitó la firma de un consentimiento informado, aprobado por el comité de ética institucional, donde se explicaron las garantías de voluntariedad, confidencialidad, privacidad y beneficencia para la participación en el estudio. Durante las entrevistas se invitó a narrar experiencias de manera cronológica, con foco en sus trayectorias personales y ocupacionales, buscando establecer consideraciones de género, en el sentido de tensiones y ejercicio de agencia, imbricando ámbitos vitales, como la educación, familia y trabajo.

Para interpretar la información se ocupó el método de análisis de contenido, a partir de la transcripción literal de las grabaciones y una categorización inductiva (Mejía, 2011) que se enfocó en los hitos significativos de sus recorridos vitales, elecciones, voluntades de acción, recursos y estrategias desde las cuales han vivido la experiencia laboral, así como el afrontamiento de tensiones de género.

\section{Resultados}

1. Socialización, capital cultural y modelo familiar en las trayectorias personales

La edad promedio de las entrevistadas es de 42 años, fluctuando entre 33 y 55. La mayoría vive en una ciudad distinta donde trabaja, y posee turnos de cuatro días -de lunes a jueves-, con jornadas de 12 horas diarias, hasta completar 45 semanales. Se desplazan en avión bajo la práctica Fly-in Fly-out (Jenkins, 2014) y tienen tiempos concentrados de trabajo en áreas geográficas remotas, sin residir cerca de las faenas. ${ }^{7}$ La mitad de las ingenieras

7 La decisión de no vivir en la ciudad cercana a las faenas se debe a que ésta carece de los servicios que tiene la capital del país y de centros educacionales de calidad. Los índices generales de calidad de vida son menores. 
metalúrgicas, químicas y de minas decidió estudiar su carrera por influencia de sus hermanos/as mayores, quienes ya habían cursado una carrera similar y les entregaron apoyo material, como la acogida en sus casas en ciudades mineras.

Sus padres no incidieron en dicha elección, aun cuando tenían oficios masculinizados en otros rubros (mecánica o transporte). La mayoría de las madres trabajó remuneradamente en actividades feminizadas (educación, administración y comercio), siendo relevante en sus elecciones educativas. Fueron estrictas con su rendimiento escolar, modelo que reproducen con sus propias hijas. La exigencia materna interpretada como impulso al desarrollo de confianza en el logro implicaría un modelamiento para tomar elecciones empoderadas, que incidiría en el modo como fueron forjando su actual identidad. "Mi mamá, sobre todo, ha sido mi gran sponsor... me empoderó harto a cumplir lo que quería... siempre tuve ese modelo, mi papá y mi mamá trabajaban" (Entrevistada 1, 34 años). ${ }^{8}$

El capital cultural que adquirieron de madres insertas en el mercado laboral, desde la perspectiva de género y ruptura de modelos dicotómicos tradicionales, les aportó sintonía con ideales como la promoción de autonomía económica, defensa de derechos de las mujeres y reconocimiento de méritos independiente del sexo. Surgieron como modelos positivos que transmitieron valores y conductas, donde la capacidad para elegir un trabajo es apreciada para el desarrollo personal, además de aprender que las expectativas requieren acciones realizadas por sí mismas, para situarse como protagonistas de sus propias vidas. Simultáneamente, la participación paterna en el campo doméstico, en especial en periodos de cesantía, incidió en la concepción de fluidez y alternancia de los roles domésticos para ambos sexos.

De las entrevistadas que tienen pareja (todas heterosexuales), la mitad trabaja en minería, confirmándose la tendencia de que mujeres mineras se emparejan con mineros (Caro et al., 2019) por la comprensión del sistema de turnos. La otra mitad es ingeniera y labora en otras industrias masculinizadas (agrícola y energía). La intensidad en la dedicación horaria que requieren los puestos altos en operación está asociada a largas jornadas, siendo un diseño que calza más con un universo de trabajo masculino (Burin, 2004). Al considerar las características del trabajo (distancia geográfica y turnos), las mujeres con pareja e hijos/as tienen acuerdos no tradicionales de distribución del cuidado infantil. Dicha negociación, con mayor participación masculina en la crianza, es un dispositivo relevante que actúa positivamente en la capacidad de agencia y elecciones laborales. La experiencia más radical ocurre con

8 Entrevista personal, 2019, Chile. 
la renuncia de una de las parejas a su carrera profesional para dedicarse exclusivamente al cuidado cotidiano de sus hijos/as y de los roles domésticos. En este caso, aun cuando el relato explicita que se trató de un arreglo espontáneo y pragmático, basado más en la mayor proyección de la carrera de la mujer que en una decisión reflexiva en materia de igualdad de género, se invirtió la tradicional división sexual del trabajo y roles, fórmula que facilitó su ascenso.

No habría ninguna manera de que hiciera lo que estoy haciendo si no fuera por mi esposo... tomó la decisión de dejar su carrera... nunca fue una decisión deliberada... fue originalmente una prueba... y luego aceptamos nuestros roles diferentes... mi confianza en él me da un relajo enorme para seguir haciendo lo que hago (Entrevistada 4, 48 años).

Las mujeres que no tienen hijos/as sostienen que las parejas son en general un obstáculo, porque no siempre son receptivos a asumir labores de cuidado (incluyendo mascotas). De los relatos se desprende que modelos familiares más igualitarios facilitan la gestión de conflictos relacionados con elecciones laborales, basadas en una dedicación más intensa a responsabilidades y jornada. Asimismo, dichos acuerdos conducirían a una menor culpa que subyace en el mandato de género de las madres trabajadoras, por la confianza en el cuidado.

2. Trayectorias educativas y agencia: excelencia y confianza en el logro de metas

En las trayectorias educativas se identificaron facilitadores para lograr metas, que siguen estando presentes en el ejercicio de sus actuales cargos. Dentro de los más transversales se encuentra el haber sido estudiantes disciplinadas, planificadas y competitivas. Siempre fueron las mejores de su clase. "Era la niña ordenada, buena, que me sacaba buenas notas, que contestaba las preguntas del profesor" (Entrevistada 1, 34 años). ${ }^{10}$ Las mujeres se convencieron de que la consecución de objetivos valorados (ser las primeras de la clase) implicaba destinar voluntad para alcanzarlos, con resultados de satisfacción no sólo por el logro en sí, sino por la aprobación de otros/as.

La competencia con sus pares hombres podría interpretarse como la confirmación de una identidad bajo la mirada evaluativa externa, como espejo que refuerza la imagen de sí mismas. Un segundo elemento que resalta es el haber desarrollado una trayectoria en los planos organizacional y dirigencial. Presidentas de cursos en secundaria o universitaria, scout y voluntariado fueron roles elegidos donde aprendieron a trabajar en equipo, el sentido de

9 Entrevista personal, 2019, Chile.

10 Entrevista personal, 2019, Chile. 
pertenencia, liderazgo, generosidad y empatía, factores que consideran valiosos para su actual desempeño y que han usado para contrarrestar tensiones y resistencias de género.

En sus recorridos formativos más recientes, algunas mujeres han adquirido o aspiran a adquirir grados académicos superiores, aunque no sea un requisito para ascender, pues ven en la continuidad de los estudios un recurso personal a desarrollar. Reconocen que nuevos aprendizajes (técnicos y actitudinales) son y serán un plus para su crecimiento laboral, lo cual les permitirá promover autonomía en sus equipos y delegar, sobre todo en una industria que además de no acoger con facilidad a mujeres en jefaturas, tiende a concentrar el poder. "Me gustaría estudiar un posgrado en minería, como para tener conocimientos técnicos ... tengo mucho todavía por crecer y aprender, estoy en una etapa que quiero crecer laboralmente" (Entrevistada 6, 35 años). ${ }^{11}$ Capacidades como la confianza en sí misma y la iniciativa, surgidas en procesos de socialización familiar y escolar, las alerta para identificar nuevos aprendizajes y contrarrestar la sensación de estar permanentemente "a prueba".

3. Movilidad social y laboral. Agencia como acciones que producen cambios

Todas las entrevistadas tuvieron una importante movilidad ascendente de clase en sus trayectorias, proceso que fue desarrollado con "sacrificios" extraordinarios y motivado por sus familias de origen, al instarles a cumplir responsabilidades desde temprana edad en contextos de escasos recursos económicos. En general, sus antecesores no eran profesionales (salvo excepciones) y carecían de recursos para financiar estudios universitarios; por eso los cursaron con becas, recurrieron a ahorros familiares o los autofinanciaron trabajando en vacaciones, lo cual para algunas tuvo efectos negativos en la salud, pero no les impidió perseverar. Los relatos reflejan además proyección de deseos paternos por movilidad vía educación. "Siempre nos decían que estudiáramos, aunque mis papás no tenían ni un peso... todo el año que juntó plata... para la universidad" (Entrevistada 5, 46 años). ${ }^{12}$

Mujeres en altos cargos perciben que los obstáculos del entorno, más que ofrecer resistencias, pusieron en escena capacidades y de alguna manera "crearon" sus vidas, desafiándose para evitar un inmovilismo perjudicial. Conformarían su identidad con la acción más que con la pasividad, por la socialización recibida y un aprendizaje que valoran de sus recorridos vitales. La mayoría inició su carrera laboral en la industria minera, donde han tenido

11 Entrevista personal, 2019, Chile.

12 Entrevista personal, 2019, Chile. 
movimientos de trabajo frecuentes. Les resulta atractivo cambiarse a nuevas oportunidades para ascender y destacar, ya sea de manera vertical (misma faena) u horizontal (otra).

En otro caso se trata de cambiarse a una faena del mismo holding, más cerca de la capital, donde "ganen tiempo" al disminuir desplazamientos. O a una más grande que explote mineral de mejor calidad, porque "ganan prestigio". O moviéndose -especialmente las que tienen hijos/as pequeños- a faenas próximas a una ciudad mediana o capital regional, porque "ganan calidad de vida". En todas estas decisiones se aprecia la capacidad de agencia como libertad de elección, al identificar un horizonte por alcanzar.

4. Tensiones de género al incursionar en espacios y posiciones no tradicionales

Estudiar una carrera "de hombres" implicó en los años ochenta o noventa cruzar un umbral de segregación de género, que las mujeres experimentaron con miedos, dolor, nervios y angustia, porque percibían que se estaban integrando "de manera forzada" a un ambiente que explícitamente las rechazaba. Después de haber vivido experiencias de acoso sexual y desvalorización por su elección vocacional por parte de profesores, han soportado acoso laboral sexista de jefaturas, lo cual las llevó a tomar decisiones de salida, perdiendo posición y salario.

Tuve un impasse con un jefe ... opté por otra empresa... ganaba mucho más allá porque era supervisora, tenía buenos beneficios, acá iba a llegar a trabajar a una empresa contratista, pero no me importó, quería mi estabilidad emocional y no trabajar con una persona que todos los días me molestaba (Entrevistada 7, 46 años). ${ }^{13}$

En casi dos décadas de trayectoria laboral, perciben que han protagonizado verdaderas revoluciones cotidianas al interior de las faenas, generando un nuevo escenario simbólico al interior de los equipos y cultura laboral, rompiendo la primacía masculina, al ingresar siendo mujeres jóvenes y que además no venían de la "cultura" minera. "Era una mujer joven, no era de la empresa... alguien que les rompía el esquema... todos me miraban con cara de pregunta, ¿qué hace esta niñita acá?” (Entrevistada 3, 38 años). ${ }^{14}$

Aun así, reconocen que los cambios culturales, específicamente en sus pares hombres, han sido lentos y escasos; más bien se tiende a reproducir rutinas que naturalizan prácticas de discriminación. Perciben que, como grupo social, las mujeres han cambiado más que los hombres, y que la fuerza de la transmisión de modelos y ejemplos de avance entre mujeres ha incentivado a

13 Entrevista personal, 2019, Chile.

14 Entrevista personal, 2019, Chile. 
otras a insertarse a la minería. Sin embargo, se sigue reproduciendo, incluso de manera naturalizada, un trato grosero y vulgar que las incomoda por su hostilidad, así como el paternalismo, una forma más velada de desigualdad, pues se les trata como si estuvieran permanentemente "expuestas al peligro", interpretándolo como exclusión y percepción de limitaciones por el hecho de ser mujer. Las acciones de sensibilización para el cambio cultural realizadas por algunas empresas (talleres o campañas comunicacionales), al ser recientes y de impacto moderado, podrían favorecer un cambio del entorno; sin embargo, todavía no logran modificaciones sustantivas en la totalidad de las organizaciones.

Completamente machista, aquí no había mujeres en operaciones, o sea soy pionera en esta planta ... éramos muy cuestionadas ... una mujer, era muy raro ... mi jefatura me decía, si vas al tranque, no vayas sola, porque es peligroso... por el hecho de estar sola y ser mujer (Entrevistada 8, 55 años). ${ }^{15}$

Todas han enfrentado situaciones de discriminación de género en sus trayectorias laborales de diversa envergadura, pero esto no ha logrado convertirse en un punto de inflexión que las hiciera o haga desistir de sus elecciones ocupacionales. Sin embargo, la tendencia ha sido afrontar individualmente los obstáculos (cambiarse de empresa), aun cuando existe conciencia de que se trata de experiencias habituales y compartidas ("éramos muy cuestionadas").

\section{Estrategias, recursos de validación y agencia frente a barreras de género}

Ante los desafíos que implica ostentar un alto cargo, se presentan matices, pues ser pionera ha tenido aspectos favorables y desfavorables. Aun cuando este hecho marca una impronta, permite aprender de sí misma y del rol, y abre caminos a otras, también es señalado como tremendamente agotador por el nivel de exposición, pues todas las miradas están puestas en su desempeño, son más vulnerables a las críticas y sanciones, y se sienten evaluadas de forma permanente. La tendencia es responder a estos obstáculos culturales sobre-demostrando sus capacidades. Otras confirman que frenaron opciones de ascenso por barreras autoobservadas, al pensar sesgadamente que necesitaban más preparación para ejercer los cargos ofrecidos o cumplir con los requisitos exigidos. Pese a ello, en la actualidad todas aspiran a ascender, no sólo por ellas, sino porque quieren dejar un legado en la historia de las mujeres en el ámbito de la minería.

En una oportunidad me ofrecieron un cargo de jefe, y en ese minuto me puse la barrera, dije no, que me faltaba un diplomado, coach... después quiero ser gerenta general y

15 Entrevista personal, 2019, Chile. 
vicepresidenta de operaciones mineras... dejar un legado a más mujeres, lo que hago bien abre las puertas a mujeres ... siempre he querido apoyar... dar charlas, ser mentora (Entrevistada 5, 46 años). ${ }^{16}$

Para enfrentar la dureza del clima relacional, los mecanismos desarrollados para contrarrestarla refieren a: la asunción de fortaleza de carácter (ser más dura y directa), pero combinando calidez y amabilidad; transmitiendo mensajes de manera clara y enérgica, aunque sean complejos e impliquen desacuerdos; visión completa de los problemas y soluciones; conciencia de los avances; madurez; autonomía; y al priorizar los aspectos humanos en la gestión del rol. Todos estos componentes contribuyen a enfrentar la hostilidad del medio, buscando dejar de ser vistas como una amenaza por sus pares masculinos.

Aprender a tener herramientas de defensa me ha permitido instalarme en el mundo minero ... me dijeron: tú sabes que acá los cargos de jefatura son sólo para hombres... He crecido en carácter, un poco más dura, ver las cosas con más claridad... he tenido una evolución, más madura, independiente, menos temerosa (Entrevistada 9, 33 años). ${ }^{17}$

El imaginario de la masculinización del rol de jefatura está presente en los relatos, en un entorno que ha tolerado liderazgos autoritarios, verticalistas y descuidados de emociones positivas en el trato. En ese contexto, creen que se espera de ellas el cumplimiento de los mismos rasgos tradicionales. Sin embargo, en la práctica, la mayoría no desea cumplirlos, o más aún, tiende a contradecirlos, desplegando atributos opuestos al estereotipo del cargo esperado, aspirando así a obtener resultados positivos tanto productivos como en el clima interno. La socialización de género marcaría su actual comportamiento, lo cual puede interpretarse como el no poder "escapar" de atributos femeninos histórica y socialmente valorados, incluso ejerciendo un rol tradicionalmente masculino. Se busca destacar desde el valor de la diferencia, más que desde una igualación mecánica, poniendo en el centro de su visión a las personas, así como los impactos y riesgos de la gestión; es una decisión que han adquirido con el tiempo, e incluso con apoyo externo, como la participación puntual en programas de liderazgo.

Se confunde un poco que tienes que golpear la mesa, ser igual de ordinaria para hablar que un hombre para que te escuchen... si me toca ser pesada, cambio un poco el tono de voz, trato de no dejar de ser amable y me entienden (Entrevistada 9, 33 años)..$^{18}$

16 Entrevista personal, 2019, Chile.

17 Entrevista personal, 2019, Chile.

18 Entrevista personal, 2019, Chile. 
Otras sostienen que el desafío de interactuar desde una posición distinta, no sólo por ser mujeres sino por realizar conductas poco tradicionales en el ejercicio de cargos de autoridad (casi todas son pioneras en la actual posición en su empresa), hace que el entorno se sorprenda y las perciba con reticencia, sin reconocer la oportunidad transformadora. "Encuentro que a mi entorno le cuesta entender qué hacer conmigo ... soy la rara... en espacios donde las personas se conocen, hay roles, cuesta meterse" (Entrevistada 10, 54 años).

Las experiencias son agenciadas, a partir de trayectorias de ascenso que se autoconstruyeron paso a paso (Tarrés, 2001b), trabajando cotidianamente desde la autoconfianza. Se observa que han buscado construir un liderazgo colaborativo, que implica hacer partícipes a otros, complementando la seguridad en sí mismas para plantear diferencias, con apertura a aprender, bajo miedo a equivocarse y asumir el riesgo de sus elecciones. Conducta disímil a lo conocido, pues señalan que en la cultura minera existe el temor a enfrentar públicamente el "no sé", y más bien entre los hombres suelen pedir ayuda por medio de pactos homosociales, siendo conservadores, pues reproducen las dinámicas relacionales que ya conocen. Para las mujeres, el nuevo ejercicio en el campo del poder implica reconocer que pueden visibilizar posibilidades de errar y con ello mejorar.

En el plano de la sociología de las emociones, una estrategia comunicacional desarrollada - nada común en ambientes mineros y que goza de poca legitimidad en espacios laborales- es la incorporación en el ejercicio del cargo de expresiones amorosas, que no tienen tapujos en demostrar, y que la asocian tanto a su pasión y vocación minera, como al valor que les otorgan a las relaciones interpersonales. En el primer caso afirman sentir amor, disposición y aptitudes por las ciencias matemáticas, la metalurgia, la estética minera y el trabajo en terreno; por lo tanto, disfrutar de su labor permite neutralizar dificultades y hostilidades.

En el segundo caso, la centralidad que ocupan las relaciones humanas, por compartir cotidianamente con otros/as, el sistema de turnos y vida en campamentos, implica darle una impronta hogareña al espacio laboral. El trato amoroso de este nuevo sujeto en la industria (mujeres jefas) sería una estrategia que busca ganar familiaridad y que se usa para confrontar la negación a priori de trabajadores a ser dirigidos por una mujer, permitiéndoles avanzar en el logro de fines personales (posicionarse) y organizacionales (conseguir las metas asociadas al cargo). Sin embargo, las mujeres son conscientes de la paradoja que conlleva desafiar identidades tradicionales y lidiar con prejuicios de género arraigados. 
Amo a mi equipo... vivo en mi trabajo, por lo tanto, se generan afectos ... desayuno, almuerzo, ceno, voy al gimnasio con la misma gente, hay un nexo que generas que es distinto a otros rubros ... pero también esperan que uno sea suavecita siempre, ese es un prejuicio, si una mujer es fuerte, choca (Entrevistada 6, 35 años). ${ }^{19}$

En un contexto de transformación social, las dimensiones de macromovimientos sociales que generan mayor reflexión acerca de los derechos de las mujeres y la igualdad de género -como biográficos, sus propias trayectorias de rupturas a lo tradicional- son generadoras de cambios a nivel identitario. Las mujeres afirman que han desarrollado mayor reflexividad, reconociendo que ellas mismas reproducían estereotipos y sesgos de género naturalizados, y que incluso eran usados como estrategias "rápidas" de integración, visiones que en la actualidad rechazan.

He naturalizado muchas cosas, que ahora con los movimientos sociales, me di cuenta que no está bien ... que están pasando a llevar... mis colegas hasta el día de hoy hacen bromas tontas, que a estas alturas no deberíamos hacer ... no me daba cuenta (Entrevistada 11,37 años). ${ }^{20}$

Como dispositivo endógeno, las más jóvenes se perciben más empoderadas en contextos homosociales, al afirmar que no las amilana su condición de mujer y al saber que están rompiendo mandatos heredados. Experiencia nada fácil. Perciben como "buena señal" participar activamente en la formación de otras mujeres, para fomentar una cultura del buen trato, propiciando flexibilidad, delegación, confianza en los equipos y evitando el microcontrol. Un dispositivo exógeno que se observó en una de las empresas que está desarrollando acciones pro-igualdad de género y que contribuye al ejercicio de un liderazgo femenino agenciado desde el empoderamiento, lo constituye las medidas de eliminación de sesgos de género presentes en políticas de promoción - por ejemplo, ascender a una mujer embarazada- que, aun cuando son minoritarias, resultan emblemáticas. Las entrevistadas no logran apreciar influencias directas del Estado en la promoción de las capacidades de agencia.

\section{Discusión}

Mujeres en altos cargos de operación minera conforman un grupo social nuevo que responde a una experiencia excepcional, lo cual hemos observado desde los prismas de género (Scott, 2008) y agencia (Sen, 1999), iden-

19 Entrevista personal, 2019, Chile.

20 Entrevista personal, 2019, Chile. 
tificando tanto transformaciones como continuidades, que se expresan de manera concomitante como tensiones. Experimentar posiciones contraculturales desde la perspectiva de género, por oficio, cargo y localización nos permite identificar mutaciones que ha sido posible observar en las biografías. Los cambios más importantes en sus itinerarios han sido: elegir una carrera masculinizada a partir del incentivo familiar y de la influencia de historias maternas emancipadas, proyectar permanentemente continuidad de estudios y ascenso laboral, desarrollar liderazgos colaborativos y con ello interrogar el liderazgo tradicional en la industria, y promover una organización de la vida doméstica y conyugal más igualitaria y democrática. Las continuidades se mantienen en la persistente resistencia cultural del entorno (subalternos y pares), que se expresa en expectativas tradicionales respecto al rol de una mujer cuando es jefa, así como en la percepción de barreras a la maternidad y la todavía escasa implementación de acciones empresariales para mitigarlas.

Como son pioneras en sus puestos, portan en sus propias trayectorias, en primera persona, el cambio paulatino de la modificación de roles al interior del mercado laboral minero, por ejercer cargos de poder antes negados, que de acuerdo a relatos de quienes llevan más de dos décadas en la industria, en primera instancia fueron empujados desde ellas mismas, y recién hace unos pocos años han comenzado a impulsar algunas empresas, presionadas por movimientos globales (corporativos y sociales) y medidas locales -como la Norma 3262- que incipientemente han contribuido a cuestionar estereotipos que frenan el ascenso de las mujeres.

Sin embargo, es el movimiento biográfico encarnado en mujeres el que más aporta a reducir discursos de inequidad. Dicha situación es experimentada conscientemente desde las mismas mujeres como una responsabilidad, pues implica procesos de choque contra barreras históricas transferidas por procesos de socialización (Héritier, 2007). Asimismo, se perciben subjetivamente como un ejemplo para otras, porque "abren puertas", interrogan "techos de cristal" (Burin, 2004) y sortean dispositivos de una cultura laboral que rechazó el ejercicio del poder protagonizado por mujeres. Aun cuando algunos mandatos de género se diluyen en ellas, sostenemos que es un tránsito que ocurre desde una visión más bien unilateral, porque se ha develado que se trata de un proceso de transformación que se sostiene y concentra más en sí mismas que en el entorno en su conjunto, incluyendo políticas empresariales y estatales.

Desde la perspectiva de las entrevistadas, entre los hombres de la industria todavía se mantienen simultáneamente posturas coadyuvantes -adherentes a objetivos de igualdad de manera explícita- y otras de 
rechazo. Al triangular con planteamientos teóricos, dicho proceso es valorado desde el lente del cambio de una dimensión individual agenciada (Nussbaum, 2014) que se constituye como confrontación al orden tradicional, aun acompañada de conflictos (Otano, 2015).

Por otro lado, se evidenció un despliegue de capacidades de agencia, comprendida ésta como el ejercicio de la libertad de hacer y lograr metas consideradas valiosas (Sen, 1985; Keleher, 2014) que, de paso, interpelaron construcciones identitarias tradicionales (Tarrés, 2007, en Olivera, 2008) al no elegir reproducir relaciones con pares o subalternos basadas en la sumisión y servicio. Experiencias en las cuales introdujeron nuevas ideas que desobedecen formas normalizadas en las relaciones sociales de género (Scott, 2008), rechazando la masculinización en el ejercicio del cargo, buscando desmarcarse de estilos autoritarios, privilegiando una conducción colaborativa, interacciones comunicativas basadas en respeto y amabilidad, el reconocimiento del valor del otro/a y la horizontalidad, que contrasta con el perfil tradicional recogido en investigaciones previas (Salinas y Romani, 2016). Desde la estética, tampoco se autoexigen modificar gestos o timbre de voz, y la mayoría mantiene íconos tradicionales femeninos como el maquillaje o las uñas pintadas, pero sin buscar estereotipar su atuendo (casco o bototos rosados).

Transitar sus trayectorias de vida permitió comprender las principales influencias culturales cotidianas que se conjugaron en la libertad para elegir (Cejudo, 2007). En el primer caso está la transferencia intergeneracional de capital cultural subjetivo (Bourdieu y Wacquant, 1992), sobre todo desde sus madres, en especial las que estuvieron insertas laboralmente. Luego aparecen hermanos/as, padres y mentores que surgieron en sus primeros empleos. Una de las "luchas" más relevadas ha sido confrontar su propia identidad actual con la de género tradicional (Tarrés, 2013) y el perfil de jefatura conocido en la industria. Se han visto a sí mismas como las "raras", primero en la universidad porque eran de las pocas que estudiaban carreras mineras; y después en las faenas por ser de las pocas que además ostentan cargos de poder siendo más jóvenes que sus pares hombres, y ejercen el cargo siendo directas y fuertes en la conducción, y simultáneamente amorosas, amables y colaborativas.

En sus entornos extralaborales también representan lo diferente en términos identitarios, por sus arreglos conyugales no tradicionales, el traslado temporal a otra zona para realizar su trabajo y dejar a sus hijos/as al cuidado de otros/as, entre otras decisiones contra normativas a una identidad y división sexual de género tradicional, perspectiva desde la cual se han valorado culturalmente formas de hacer, experiencias y representaciones (Fraser, 2011). 
Si bien se trata de historias basadas más bien en méritos personales, aun así están vinculadas a procesos de transformación más de tipo macro (Tarrés, 2007 en Olivera, 2008), como la eliminación de restricciones legales para las mujeres en minería subterránea y la influencia de procesos de globalización culturales que inciden en declaraciones empresariales y en la definición de iniciativas gubernamentales (Norma 3262). Los cargos que ostentan son posiciones ganadas desde una mirada de género, clase y edad; poniéndose en juego la interseccionalidad (Expósito, 2012) como categoría explicativa para comprender el poder de contrarrestar desigualdades que se cruzan a través del empuje de estrategias de posicionamiento, las cuales - desde diversas variables y no sólo la del género- desafían el statu quo.

En las estrategias de validación (De Certeau, 2000, citado en Madero y Mora, 2011), entendidas como capacidades de agencia (Sen, 1999) y recursos desplegados en contextos hostiles y de baja tolerancia al liderazgo femenino, el estudio recoge diversas acciones desarrolladas para sortear dificultades en el ejercicio de la posición alcanzada, como sobre demostrar capacidades (Caro et al., 2019) en el proceso de legitimación, pudiendo interpretarse como injusticia de género, pues es una demanda específica focalizada en ellas, no transversal al conjunto de las jefaturas; ser colaborativas para no perfilarse como una amenaza a sus pares; complementar seguridad en las competencias técnicas con la capacidad de preguntar; y rechazar un estilo confrontacional que se validó de manera subjetiva largamente en el pasado y cuestionado en la actualidad desde modelos modernos de liderazgo. En dichas estrategias se consignan algunas que son parte del estereotipo femenino, pues se asocian a la afectividad o emociones "cálidas" (Burin, 2004), como el amor y la horizontalidad. Sin embargo, otras se vinculan con su opuesto, emociones "frías" (Burin, 2004), como exigir eficacia en la tarea. Se trataría entonces de perfiles transicionales, que presentan muchas veces conflicto por compatibilizar los dos tipos de vinculación -predominio afectivo y racional- (Burin, 2004), pero que aparecen latentes tras la búsqueda de metas personales y laborales.

Como limitaciones y desafíos del actual trabajo y posibilidades de ampliación de miradas y articulación de interpretaciones, se proyecta recoger las percepciones de otros actores de la industria, como hombres en altos cargos y en posiciones de subordinación laboral, en torno a las capacidades de agencia y tensiones de género que experimentan mujeres en altos cargos. 


\section{Conclusiones}

La participación excepcional de mujeres en cargos de dirección en minería, analizada desde una dimensión cualitativa y bajo el lente de las categorías de género, como relaciones significantes de poder (Scott, 2008) y agencia (Sen, 1999), permite aproximarnos a la comprensión de elementos simbólicos que se pondrían en juego en un proceso de transformación cultural en curso, y que si bien contiene trasgresión de lo normativo - posiciones e identidades tradicionales-, debe ser examinado en sus paradojas y con cautela temporal. Aun así, sostenemos que, en términos amplios, interroga al orden de género que opera como dispositivo de producción de subjetividades y actúa constriñendo a los sujetos y cuerpos en un entramado de relaciones de poder (Rebolledo y Valdés, 2018) tradicional. Realidades encarnadas en mujeres que ocupan altos cargos en minería, que resultan extraordinarias, pueden ser reconocidas como la punta de lanza de un devenir que pareciera no tener retorno cultural (Díaz, 2014) e institucional, pues es refrendado por programas gubernamentales y empresariales que la misma industria propicia, y por nuevas representaciones que impulsan las mujeres desde sus trayectorias y experiencias cotidianas, que respaldan procesos de cambio. Éstos se articulan con significados que circulan desde lo social y permiten aportar a la comprensión de horizontes de transformación de las relaciones de género en un mercado laboral homosocial, el cual ha mostrado la permanencia de resistencia al cambio y con prácticas de discriminación de género que aún perviven.

En relación con las preguntas e hipótesis, concluimos que las principales tensiones de género que enfrentan mujeres en altos cargos en minería, y que podrían ampliarse a otras industrias masculinizadas, se presentan como: a) conflictos en los roles familiares, b) en el poder y reconocimiento, c) en las representaciones culturales del medio y d) la redefinición de identidades. En el primer ámbito, la maternidad, crianza y cuidado siguen siendo una de las principales fuentes de tensión exacerbada por las características de la actividad (distancia, turnos, baja conectividad) y alta responsabilidad del cargo, que expresada como conflicto paradojalmente aparece con mayor intensidad en quienes aún no son madres bajo el señalamiento del "fantasma de la maternidad", nueva metáfora que se suma a la del "muro de la maternidad" que la literatura ha consignado (Minerals Council of Australia, 2013).

En segundo lugar, se observan tensiones de género por el modelo histórico de ejercicio del poder masculino y nuevos atributos que posicionan a las mujeres, donde se imbrican elementos de la feminidad tradicional portados por la socialización y nuevas subjetividades femeninas (Jenkins, 2014). El 
modelo de concentración del poder y liderazgo heredado, vertical y autoritario, se tensa con improntas colaborativas y horizontales desplegadas por las mujeres que, en un contexto inicial de rechazo y exclusión, hoy han transitado a una vigilancia velada, pues están más expuestas al error y se autoperciben estando siempre "a prueba". En tercer lugar, las tensiones de género que se generan de las representaciones culturales del entorno han sido experimentadas como acoso sexual y sexista del pasado (universidad o anteriores empleos), ahora como incomodidad y malestar frente a la naturalización del trato grosero y denigrante, a la exigencia por masculinizarse en el ejercicio del rol (gestual o verbal), porque es lo que se espera y conoce, al paternalismo como muestra de sexismo benevolente, y por ser vistas como una amenaza, pues su sola presencia introduce cambios a una cultura homosocial. Las acciones de las empresas, algunas incididas por iniciativas gubernamentales, como instancias de sensibilización, han sido puntuales (no permanentes) y sus efectos son moderados. Finalmente, la tensión identitaria se manifiesta en la percepción de ser las "raras" en el entorno laboral, pero también fuera de él, lo cual pareciera introducir modificaciones en sus perfiles autoobservados, sin otorgarle una connotación positiva ni negativa. De esta expresión se recoge la idea de movimiento biográfico que implica reacomodos en este caso individuales, donde conviven cambios parciales y quiebres, pero que podría proyectar negociaciones de significados a un nivel macrosocial, sin pretender mirar las identidades de las mujeres en altos cargos en un sentido homogéneo.

Respecto a los recursos y estrategias desplegados, interpretados desde el pivote de la agencia, concluimos que tienen preeminencia en una dimensión más bien individual que colectiva, aunque con proyección intencionada hacia lazos de solidaridad, toda vez que sostienen pretender en el futuro dejar un legado y transferir aprendizajes a otras mujeres. Desde lo microsocial, los recursos personales tienen un recorrido vivencial, intergeneracional e interseccional (con clase y edad). Desde una mirada histórica intratrayectoria, la investigación identificó recursos anidados desde la experiencia educativa primaria y luego superior -basada en la disciplina, excelencia, competencia y participación en roles dirigenciales y de ayuda social-, atributos que se fueron fraguando después en sus itinerarios formativos y laborales; asimismo, recogieron de sus modelos parentales flexibles la búsqueda de arreglos conyugales no tradicionales e igualitarios.

Desde una perspectiva histórica intergeneracional es principalmente el capital cultural transferido por sus madres de orientación emancipatoria, acompañado de facilidades pragmáticas otorgadas por hermanos/as y motivación paterna por la movilidad social, lo que incidiría en biografías 
agenciadas, produciéndose un cambio en la producción de patrones sexuados de elección y comportamiento, que incidió en una vocación y trayectoria no tradicional desde la perspectiva de género. Con base en la mirada interseccional (Expósito, 2012), considerando las características estructurales e históricas de la cultura de la industria - dureza y aridez del entorno y complejidad práctica-, el origen de clase de las entrevistadas (familias de escasos recursos, con experiencias laborales tempranas) y la prematura claridad en su proyecto de ascenso social inciden positivamente en el despliegue de recursos personales para enfrentar barreras de clase, edad y género, forjándose atributos como resiliencia y capacidad para enfrentar adversidades ante los prejuicios por ser mujeres, no provenir de familias profesionales y ser más jóvenes que sus subalternos.

A modo de síntesis, las estrategias deliberadas que usan en la actualidad las mujeres en altos cargos para contrarrestar tensiones y barreras de género en minería son las siguientes: a) los arreglos familiares-conyugales más igualitarios les permiten disminuir la culpa, trabajar con mayor confianza y aumentar la capacidad de responder afirmativamente al ascenso laboral; b) seguir y proyectar continuidad de estudios para contrarrestar la percepción de ser las "recién llegadas" y "estar a prueba"; c) demostrar una identidad basada en la acción, éxito y logros tanto técnicos como actitudinales para sortear el rechazo de la cultura a ser dirigidos por una mujer; d) como estrategias subjetivas de defensa a la hostilidad buscan conjugar fortaleza de carácter y seguridad en sí mismas con trato amoroso, sin descuidar aspectos humanos del proceso productivo; e) finalmente se oponen a exigencias de masculinización del cargo desde una impronta autoritaria, valorando el aporte diferencial desde un estilo de liderazgo colaborativo.

Confirmamos el planteamiento teórico que sostiene que las capacidades de agencia desplegadas por jefaturas mujeres no significa que dejen de persistir condiciones de desigualdad estructural de género a corregir (Sen, 2009), pues en la sociedad se sigue perpetuando un orden de género donde los hombres, en términos generales, detentan mayor poder y privilegios (Rebolledo y Valdés, 2018); cuestión que las mismas entrevistadas han validado y que se mantiene como conflictos de género en minería, personificados en subalternos y pares hombres. Sin embargo, aun considerando dichas desigualdades como parte del entramado relacional y cultural, las elecciones formativas y laborales de mujeres en altos cargos evidencia ejercicios de agencia, entendida como libertad de elección y desarrollo de acciones para el logro de dicha agencia (Keheler, 2014), cuestionando el orden de género, confirmándose la hipótesis general de la investigación. 
Extender el uso de la teoría de agencia a la realidad empírica estudiada nos permite confirmar la hipótesis específica del artículo, pues trayectorias educativas que sortean discriminaciones de género tempranamente, proyecciones de continuidad de estudios, modelos marentales disruptivos, arreglos familiares más igualitarios y estilos de liderazgo colaborativos y eficaces inciden positivamente en las posiciones de poder de las mujeres en minería. Así se sostiene la posibilidad de ser sujetos sociales agenciados que empujan y, al mismo tiempo, son envueltas en procesos de cambio movilizados, en primera instancia individuales, con potenciales efectos colectivos, aun parciales.

Lo anterior constituye un desafío societal, que en el presente tiene más alcances micro, en sus propios itinerarios, y puede ser iniciador de cambios sociales macro. Se confirma que la experiencia incipiente de mujeres en altos cargos ejemplifica tensiones civilizatorias (Valcárcel, 2009), en el sentido de trascendencia, fijando posibilidades para otras mujeres, pues comienzan a constituirse modelos femeninos antes inexistentes, a propósito del empuje desde las propias mujeres, más que facilitados necesariamente por el entorno, ya que las iniciativas de las empresas son aún incipientes y las del Estado de bajo alcance. El desafío es seguir profundizando en la comprensión del devenir de estos procesos de transformación en curso.

\section{Referencias}

Ayala, Elizabeth (2012), "Mujer y minería: situación de la industria chilena”. Tesis para optar al grado de Magíster en Gestión de Personas, Facultad de Economía y Negocios, Chile: Universidad de Chile.

Angelcos, Nicolás e Isola, Enzo (2017), "Violencia y táctica en los procesos de integración de las mujeres a la minería del cobre en Chile", en Psicoperspectivas. Individuo y Sociedad, núm. 16, Chile: Universidad Católica de Valparaíso.

Bourdieu, Pierre y Wacquant, Lois (1992), Respuestaspor una antropología reflexiva, México: Grijalbo.

Burin, Mabel (1990), El malestar de las mujeres: la tranquilidad recetada, Argentina: Paidós.

Burin, Mabel (2004), "Género femenino, familia y carrera laboral: conflictos vigentes", en Revista Subjetividady Procesos Cognitivos, núm. 5, Argentina: Universidad de Ciencias Empresariales y Sociales.

Campos, Leticia et al. (2016), "Desde la educación superior abriendo espacios de discusión y reflexión para la participación e inserción igualitaria del género femenino en el sector minero", en XXX Congreso de Educación en Ingeniería, Chile: Universidad de la Frontera.

Caro, Pamela et al. (2019), Inclusión sustentable de mujeres en industrias masculinizadas. La Mineria en Chile, Chile: Andros.

CCM (Consejo de Competencias Mineras) (2019), Fuerza Laboral de la Gran Minería chilena 2019-2028. Diagnóstico y Recomendaciones, Chile: Consejo de Competencias Mineras. 
Convergencia Revista de Ciencias Sociales, vol. 28, 2021, Universidad Autónoma del Estado de México

Cejudo, Rafael (2007), “Capacidades y libertad. Una aproximación a la teoría de Amartya Sen”, en Revista Internacional de Sociología, vol. 65, núm. 47, España: Consejo Superior de Investigaciones Científicas.

De Sousa Santos, Boaventura y Rodríguez, César A. [eds.] (2007), El derecho y la globalización desde abajo. Hacia una legalidad cosmopolita, España: Anthropos.

Díaz, Estrella (2014), "Mujeres en trabajos de hombres: segregación ocupacional y condiciones laborales en los sectores minería y construcción”, en Cuadernos de investigación, núm. 49, Chile: Dpto de Estudios, Dirección del Trabajo.

Elias, Norbert (1990), La sociedad de los individuos, España: Península.

Expósito, Carmen (2012), “¿Qué es eso de la interseccionalidad? Aproximación al tratamiento de la diversidad desde la perspectiva de género en España”, en Revista Investigaciones Feministas, núm. 3, España: Universidad Complutense de Madrid.

Forero, Yésica (2012), "Las capacidades y oportunidades de las mujeres como protagonistas integrales en el desarrollo humano", en Criterio Jurídica Garantista, núm. 7, Colombia: Universidad Autónoma de Colombia.

Fraser, Nancy (2011), “¿De la redistribución al reconocimiento? Dilemas de la justicia en una era 'postsocialista”, en Fraser, Nancy et al. [coords.], Dilemas de la justicia en el siglo XXI: género y globalización, España: Ediciones UIB.

Héritier, Françoise (2007), Masculino/femenino II. Disolver la jerarquía, Argentina: Fondo de Cultura Económica.

Instituto Nacional de Estadísticas (2018), Encuesta Nacional del Empleo, semestre octubre, noviembre y diciembre, Chile: Instituto Nacional de Estadísticas.

Jenkins, Katy (2014), “Women, mining and development: An emerging research agenda”, en The Extractive Industries and Society, vol. 1, núm. 2, Países Bajos: Elseiver.

Jiménez, Catalina et al. (2014), "Género, trabajo y subjetividad: el lugar de la mujer en la minería”, en Revista Persona y Sociedad, vol. 28, núm. 3, Chile: Universidad Alberto Hurtado.

Keleher, Lori (2014), “Sen and Nussbaum: Agency and Capability- Expansion”, en Ethics and Economics, vol. 11, núm. 2, Canadá: Centre de Recherche en Éthique de l'Université de Montréal.

Kornblit, Ana Lía (2004), "Historias y relatos de vida: una herramienta clave en metodologías cualitativas”, en Kornblit, Ana Lía, Metodologías cualitativas en ciencias sociales, Argentina: Biblos.

Lahiri-Dutt, Kuntala (2012), "Digging women: towards a new agenda for feminist critiques of mining”, en Gender, Place \& Culture: A Journal of Feminist Geography, vol. 19, núm. 2, Reino Unido: Routledge.

Leiva, Esteban (2015), Poder, agenciay empoderamiento, Argentina: Congreso Latinoamericano de Teoría Social.

Leiva, Sandra y Comelin, Andrea (2015), "Conciliación entre la vida familiar y laboral: Evaluación del programa IGUALA en una empresa minera en la región de Tarapacá”, en Polis, Revista Latinoamericana, vol. 14, núm. 42, Chile: Universidad de Los Lagos.

Madero, Ignacio y Mora, Claudia (2011), "Capital Social e Inclusión Laboral. Una aproximación a las trayectorias de ascendencia laboral de migrantes peruanos en Chile”, en Polis, Revista Latinoamericana, vol. 10, núm. 29, Chile: Universidad de Los Lagos.

Mayes, Robyn (2019), "Mobility, temporality, and social reproduction: everyday rhythms of the 'FIFO family' in the Australian Mining Sector", en Gender, Place \& Culture, 
vol. 27, DOI: 10.1080/0966369X.2018.155455. Disponible en: https://www. tandfonline.com/doi/full/10.1080/0966369X.2013.769430?scroll=top\&needAcce ss=true [enero de 2020].

Mejía, Julio (2011), "Problemas centrales del análisis de datos cualitativos", en Revista Latinoamericana de Investigación Social, vol. 1, núm. 1, México: Universidad La Salle.

Minerals Council of Australia (2013), Workforce Gender diversity review White paper. "It's Not Just a Program", Australia: Minerals Council of Australia.

Nussbaum, Marta (2014), Emociones politicas. ¿Por qué el amor es tan importante para la justicia?, España: Paidós.

Olivera, Rocío (2008), "Los significados del trabajo femenino en el mundo global. Propuesta para un debate desde el campo de la cultura y las identidades laborales", en Estudios Sociológicos, vol. 26, núm. 77, México: El Colegio de México.

Otano, Guillermo (2015), "La libertad como relación social: una interpretación sociológica del enfoque de las capacidades de Amartya Sen”, en Revista Iberoamericana de Estudios de Desarrollo, núm. 4, España: Universidad de Zaragoza.

PNUD (2019), Una década de cambios hacia la igualdad de género en Chile (2009-2018): avances y desafíos, Chile: Programa de Naciones Unidas para el Desarrollo.

Rebolledo, Loreto y Valdés, Ximena (2018), "Género y orden social: dificultades para implementar relaciones de género igualitarias en la vida cotidiana”, en Revista Anales, núm. 14, Chile: Universidad de Chile.

Riquelme, Verónica (2011), ¿La maternidad castigada? Discriminación y malos tratos, Chile: Dirección del Trabajo, Departamento de Estudios.

Rubin, Gayle (1975), El tráfico de mujeres. Notas sobre una economía politica del sexo, Estados Unidos: Monthly Review Press.

Ruiz, José (2003), Metodología de la investigación cualitativa, España: Universidad de Deusto.

Salinas, Paulina et al. (2012), "Discursos sobre la discriminación de género en los trabajadores mineros del norte de Chile", en Revista Atenea, núm. 505, Chile: Universidad de Concepción.

Salinas, Paulina y Cordero, Camila (2016), “Transformación de los liderazgos en la minería: gestión estratégica para incorporar mujeres en la industria”, en INNOVAR. Revista de Ciencias Administrativas y Sociales, vol. 26, núm. 61, Colombia: Universidad Nacional de Colombia.

Salinas, Paulina y Romani, Gianni (2016), "Hegemonía masculina, freno en equipos mixtos en la minería chilena”, en Revista Mexicana de Sociología, núm. 78, México: Universidad Nacional Autónoma de México.

Scott, Joan (2008), Género e historia, México: Fondo de Cultura Económica.

Sen, Amartya (1985), “Well-being, Agency, Freedom: The Dewey Lectures 1984”, en Journal of Philosophy, vol. 82, núm. 4, Estados Unidos: Universidad de Columbia.

Sen, Amartya (1999), Desarrollo y libertad, España: Planeta.

Sen, Amartya (2009), La idea de la justicia, México: Taurus.

Soraire, Analía et al. (2013), "Mineras. Trabajar y habitar en las minas. Un análisis desde la antropología del trabajar, la producción social del hábitat y la perspectiva crítica de género", en Revista de Antropología Experimental, núm. 13, España: Universidad de Jaén. 
Tarrés, María Luisa (2001a), "De la necesidad de una postura crítica en los estudios de género", en Revista de Estudios de Género: La Ventana, vol. 2, núm. 13, México: Universidad de Guadalajara.

Tarrés, María Luisa (2001b), Rupturas, campos de acción y construcción de identidades, Ponencia Latin American Studies Association, Estados Unidos: Latin American Studies Association. Disponible en: https://es.scribd.com/document/453898966/ TarresMariaLuisa-pdf [enero 2020].

Tarrés, María Luisa (2013), “A propósito de la categoría género: leer a Joan Scott”, en Estudios Sociológicos, vol. 31, núm. 91, México: El Colegio de México.

Valcárcel, Amelia (2009), Feminismo en el mundo global, España: Cátedra.

Vela, Fortino (2004), "Un acto metodológico básico de la investigación social: la entrevista cualitativa”, en Tarrés, María Luisa [coord.], Observar, escuchar y comprender. Sobre la tradición cualitativa en la investigación social, México: Facultad Latinoamericana de Ciencias Sociales México y El Colegio de México.

Pamela Caro Molina. Doctora en Estudios Americanos, mención en Estudios Sociales y Políticos, Instituto de Estudios Avanzados, Universidad de Santiago de Chile. Directora del Centro CIELO Universidad Santo Tomás, Santiago (Chile). Líneas de investigación: mercados de trabajo, estudios de género, familia y trabajo. Publicaciones recientes: Armijo, Lorena, Caro, Pamela y Román, Helena (2020), "Agencia individual y colectiva en dirigentas sindicales de la industria de la minería en Chile: un análisis articulando perspectiva de las capacidades y género", en Revista Izquierdas, vol. 49, Chile: Ariadna Ediciones; Caro, Pamela, Armijo, Lorena y Román, Helena (2020), "Cuerpos de mujeres, significados de género y límites simbólicos en la gran minería en Chile", en Polis, Revista Latinoamericana, vol. 55, Chile: Universidad de Los Lagos; Caro, Pamela (2019), "Childhood in the rural areas of Chile, intergenerational and gendered perspectives", en Revista Childrens Geographies, vol. 17, núm. 2, Reino Unido: Routledge Journals, Taylor \& Francis Ltd.

Lorena Armijo Garrido. Doctora en Sociología, Universidad Complutense de Madrid. Investigadora de la Universidad Santo Tomás, Santiago (Chile). Líneas de investigación: mercado de trabajo, desigualdad de género y organización social del cuidado. Publicaciones recientes: Armijo, Lorena (2019), "Los discursos de legitimación sobre el trabajo de las madres trabajadoras en España”, en Cuadernos de Relaciones Laborales, vol. 37, núm. 2, España: Universidad Complutense de Madrid. 
Helena Román Alonso. Doctora en Sociología, Universidad de Salamanca; investigadora de la Universidad Santo Tomás, Santiago (Chile). Líneas de investigación: identidad social, género y trabajo, y juventud. Publicaciones recientes: Caro, Pamela, Román, Helena y Armijo, Lorena (2019), "Cuerpos de mujeres, significados de género y límites simbólicos en la gran minería en Chile", en Polis Revista Latinoamericana, vol. 55, Chile: Universidad de Los Lagos; Caro, Pamela, Armijo, Lorena y Román, Helena (2019), "Agencia individual y colectiva en dirigentas sindicales de la industria de la minería en Chile: un análisis articulando perspectiva de las capacidades y género", en Revista Izquierdas, vol. 49, Chile: Ariadna Ediciones; Caro, Pamela; Ilabaca, Paola; Román, Helena; Armijo, Lorena; Celis, Karem; Meyer, Lesly (2019), Inclusión sustentable de mujeres en industrias masculinizadas. La mineria en Chile, Chile: Andros. 